\title{
Emotional support and diverse treatments: The role of social workers during the COVID-19 pandemic- Hasharon Corona hospital experience
}

\author{
Avigail Rot-Domen ${ }^{1}$, Sima albo ${ }^{1}$, Keren Ashry ${ }^{1}$, Lihie Eisenberg ${ }^{2}$, Jlal Bathish ${ }^{2}$, Aliza Zeidman ${ }^{2 *}$ \\ ${ }^{1}$ Social Work Unite, Hasharon Hospital, Rabin Medical Center, Sackler School of Medicine, Tel Aviv University, Israel \\ ${ }^{2}$ Internal Medicine B-Corona B Department, Hasharon Hospital, Rabin Medical Center, Sackler School of Medicine, Tel Aviv University, Israel \\ Address for correspondence: \\ Aliza Zeidman, Head of Internal Medicine B - Corona B Department, Hasharon Hospital. KKL 7, St Petah Tikva , Israel. Phone - 97239372363 , \\ Email- alizaz@clalit.org.il
}

Submitted: 28 June 2020

Approved: 12 July 2020

Published: 15 July 2020

How to cite this article: Zeidman A, Rot-Domen A, Albo S, Ashry K, Eisenberg L, Bathish J. Emotional support and diverse treatments: The role of social workers during the COVID-19 pandemic- Hasharon Corona hospital experience. G Med Sci. 2020; 1(3): 001-006.

https://www.doi.org/10.46766/thegms.virology.20070101

Copyright: () 2020 Aliza Zeidman, Avigail Rot-Domen, Sima albo, Keren Ashry, Lihei Eisenberg, Jlal Bathish. This is an open access article distributed under the Creative Commons Attribution License, which permits unrestricted use, distribution, and reproduction in any medium, provided the original work is properly cited.

\section{Introduction}

COVID-19 is one of the greatest global health pandemic crises of the 21th century, affecting millions of people worldwide. Neither known treatments nor vaccines currently exist for the severe acute respiratory syndrome coronavirus 2 (SARS-CoV-2), as a result, the disease is spreading throughout the world causing health and economic consequences [1].

The first Israeli COVID-19 positive patient was diagnosed in February 2020 [2]. Hasharon hospital was then reopened as the first COVID-19 hospital in Israel [3]. In addition to the characteristic physical signs and symptoms of the disease, these patients had to confront many emotional challenges, including isolation from their loved ones and huge mental stress while coping with a new disease with limited medical information on its clinical course and available treatments. In this article, we discuss the unique challenges for the social workers in the COVID-19 departments, diverse interventions that were taken during this period, including emotional support and treatments involving the patients, their families and the medical staff.

Hospitalization, in general, is a distressing situation for both the patients and their families. They have to cope with the unfamiliar new reality that was enforced on them following their hospitalization; with many changes followed in their private, familial and social life aspects as consequences of their hospitalization. As social workers we aim to help the patients and their families during their hospitalization period. We wish to help them adjust to the disease, unfamiliar environment and distresses, in the most possible and effective way. It is also extremely important for us to promote the social welfare of the patients and their families. 
The routine task of social workers in hospitals is mainly to help the patient cope with his/her emotional, familial, behavioral and functional difficulties rising during hospitalization. Furthermore, when the hospitalization period is over and the patient is discharged from hospital, we discuss with each patient and families, the appropriate discharge destination (e.g. Their home or nursing home or rehabilitation institution), in accordance to the welfare rights and the different hospital services they deserve during hospitalization period and at discharge [4].

COVID-19 global pandemic brought unknown challenges for us as social workers. It is characterized by tremendous insecurity feelings for the patients, their families and the medical teams [5]. Though we had many years of experience with other pandemics and other national emergencies in Israel, we have learned that this pandemic does not resemble any situation we have dealt with or practiced before. Everyday brought new information and while we consistently learned new things each day, we established the social workers guidelines for COVID-19 patients to be used by all the social-workers in Hasharon Corona hospital.

\section{A novel therapeutic setting}

Due to the highly contagious nature of the SARS-CoV-2 virus, we were instructed to avoid face-to-face meeting with our patients. Moreover, family members or any visitors were not allowed to visit the patients. Indeed, a new therapeutic environment was created in the hospital, unfamiliar for both the patients and the medical teams. Therefore, it was crucial to provide information, and mediate between the patients, their families and the medical team.

\section{Communication with the patients during the COVID-19 pandemic: a remote commu- nication era}

Normally, the social workers meet the patients and their families in-person. This face-to-face interaction is important to the social workers-patients relationship, as they promote an intimate platform for conversion and allowing the patients to feel comfortable to discuss their personal issues. Unfortunately, during the COVID-19 pandemic, the main communication was remote and digital: we suddenly begin to use phone calls, video calls, and computer screens in order to communicate with each other. This remote communication was extremely different from the intimate, inperson communication, we were familiar and used before. The communication with the patients through the computer screens emphasized the physical distance between us and the patients. Yet, we had to find out how could we gain patients trust, create successful relationships, and provide safe and intimate platform for conversion, despite not being able to meet them personally [6].

The novel remote communication setting and the subsequent termination of face-to-face interactions could create frustration, dissatisfaction and antagonism for both the patients and the medical teams [7] and efforts were made to overcome these obstacles and to preserve an intimate environment for conversions as possible; we had to see and treat the patients as there were no computer screens crossing between us, try and demonstrate empathy by emphasizing voice intonations, make sure that the patient knows that the social worker is available for them to any question or conversion as needed and show true concern in their well-being and our significant motivation and dedication to improve it, during the hospitalization and afterwards [8].

\section{Accessibility and availability of treatments}

Therapeutic changes occurring during the COVID-19 pandemic were expressed not only by the nature of the treatments but also by their availability and accessibility. Prior to the pandemic, we were involved in the treatment of specific hospitalized patients with specific indications. During the pandemic, we met and treated all the hospitalized positive COVID-19 patients and their families. We followed them from first day of hospitalization and during the whole hospitalization period. The illness and hospitalization period were followed by huge distress, confusion and uncertainty for both the patients and their families. We had to stretch our boundaries, be creative and available at all times. In order to provide the maximal availability during all hours of the day, the patients received our personal phone numbers. This gesture had tremendous consequences on our relationships with the patients, as we aimed to treat and take care of them not only on our formal working hours, but throughout the day. Unique situations that we have never experienced before aroused including coordination of food and personal equipment delivery from the families to the patients, organising breast milk pump for young breastfeeding mother, and delivering her breast milk bottles to her baby at home because only medical staffs were allowed to enter the hospital. Traditional and religious challenges such as prayer books and bibles were resolved by donations and getting access to virtual bible books. 


\section{COVID-19 pandemic and ethical dilemmas}

During the pandemic we experienced different ethical dilemmas too. One example is a situation in which the social worker himself is a person with high-risk profile for infection with the virus. On one hand, there is a care-giver who needs to take extra precautions for being at higher risk for the disease and on the other hand there is the patient which is in danger for mental health deterioration. Indeed, one of the patients was intubated for couple of days during his hospitalization period, and when he was extubated and woke up, he was very frightened. The social worker talked with him on the phone and following the patient's depressed feelings, the social worker considered meeting the patient face-to-face. After consulting the medical staff and the fact that she was at higher risk for contamination, she sadly decided to keep their communication by phone calls and be helped by the medical staff. In some manner, by her decision she might prioritize her well-being over the patient's well-being but if she got infected and sick, she will not be able to help this patient and others [9].

\section{Emotional aspects of the positive COVID-19 patients}

The majority of the positive COVID-19 patients had asymptomatic or mild to moderate disease and therefore recovered from the disease with no hospitalization, or with only short-period hospitalization. However, there was no association between the disease severity and the hospitalization period to the emotional feelings and expression of the patients, vulnerability and helplessness. Mental strength and mostly mental flexibility, with the ability to cope with the changing reality, were necessary elements for coping with the isolation, space reduction and in some cases personal losses [10].

The main emotional findings following hospitalization in the Corona wards includes: Need to adapt to new reality isolation in a Corona department, strange and remote environment, without the support of family or friends. Concern and fear for self-health disease complications and family members being infected as well. Dealing with guilty feeling, in the case where the patient has infected others, for instance, a grandmother who was anxious of the possibility that she was the one who infected her baby grandchild, A daughter who was anxious of the possibility she infected her 90 years old mother, and a soldier who was in the middle of training course in the army, were among these patients. For some of them the feeling of guilt was unbearable. Fear of society anger and frustration reactions and social shaming as for each positive COVID-19 patient epidemiological details were published in the public health media including address and places the patient visited prior two weeks before. Loss of privacy, during hospitalization with all the digital media, computers and screens in hospital and afterwards in Corona hotels. Overflow of negative emotions, as the hospitalization period could trigger and aggravate earlier emotional states. Loneliness and meaningless feelings evoked by the isolation from family and friends potentially leading to suicidal thoughts and threat to life expectancy. Frustration and anger evoked by the strong desire of each patient to return to natural environment and regular habits. Sometimes, patients expressed anger toward the social workers when they were discharged to COVID-19 hotel for further isolation instead of their own homes. The transition to a new place that requires another adjustment provoked anxiety feeling that were expressed by anger and accusation.

The fear of losing control and helplessness were key feeling during hospitalization. The combination of poor physical status that made them dependant on others, along with the unavailability of immediate response, caused distress and even despair. Some events were experienced as meaningful and significant, although prior to the pandemic they would have been experienced as minor effect. For example, a patient who was admitted to the hospital at the middle of the night shared with us the unpleasant feelings when she had to be transferred latter to a Corona hotel rather than her home. The patient described the experience as feeling like "a package that was delivered between hands and not being seen as a human being". A major cause for frustration, anxiety and anger was the serial number the patients received on admission, especially among the holocaust survivors, which made them feel like losing their identity and self being.

Sleeplessness was common accompanied by fear and anxiety which further deteriorated while sleeping and concerns that no one can notice through the cameras and the computer screens. The isolation brought severe sense of claustrophobia, for traveling, performing physical activity and participating in social meetings were forbidden prior to hospitalisation and were accentuated during hospitalization period. 


\section{Hospital discharge planning ahead process}

In routine and normal days, the social work team is involved in planning discharge for patients according to their medical situation, activity, and independence, mental state and family resources. We offer the patient and families options like going back home with caregiver or going to nursing homes or hospitals for chronic illness or mental disability [11]. At Corona times the whole decision-making process has been changed: The patient could not go back home unless he/she had two consecutive negative PCR tests which led to the situation of a totally healthy patient who was still PCR positive and still has to stay for isolation in hospital. A month later, the option of Corona hotel and home isolation were available but still in the first days of hospitalization there was no option for discharge and the whole process needed approval of the ministry of health department. The patients needed to be encouraged and we had to support the patients during their difficulties that emerged in early hospitalization days. As long as the patient did not have a negative COVID-19 result he could not be discharged to go to his home. Gradually, there were more discharge destinations options in the country for COVID-19 patients, and the appropriate discharge destination for each patient was selected based on the patient's medical condition, comprehension and compliance to treatment, alongside his family and living conditions. The leading consideration at this point was to prevent disease spreading in families, care givers and community. A patient without any support system at his home could not be discharged to go to home as there was no one who will take care of his needs. In the cases of patients having large families with multiple members, we examined whether there are sufficient conditions for home-isolation. Young and independent patients preferred to be discharged to further follow-up and treatment in the COVID-19 hotels. Patients who could not be discharged to go to their own home or hotels, mainly the older patients, had to be discharged to a temporary home-nursing setting or stay in the hospital until they had a negative COVID-19 test result. The release from the hospital to the different discharge destinations was often accompanied by dilemmas related to the 'Patient's rights'. In some cases, in contrast to the obvious nature of social work, we had to use a 'paternalistic' approach, where we took the decisions for the patients. While some patients expressed their desire to be released to their homes or to another familiar setting, sometimes we had to release them to other discharge destinations against their will, in order to prevent the disease spreading. In contrast to routine days, where the decision of hospital discharge destination was made in a full collaboration with the patient, during the pandemic, we could not always allow the patients to return to their own homes. From a public health perspective, the ministry of health instructions for positive COVID-19 patients were to maintain isolation from their families and their homes as long as their COVID-19 tests results were positive. However, in some cases, we decided to supersede these rules, out of thoughtfulness of the familial and mental state of the patients, for example, parents to young children who otherwise were left alone at home, or young breastfeeding mother that was otherwise separated from her baby. These patients were discharged to their homes although they did not have a negative COVID-19 test result, in order to prevent severe distress for the patients and their young family members. Those exceptional homedischarge approvals were precedents for another home-discharge for positive COVID-19 patients in the future. By doing so, we were able to restore autonomy and collaboration feelings for the patients and their families.

\section{End of life}

Throughout the history and in all cultures, death has been considered as a significant event, which has a major impact on both personal and family-social aspects. The attitude toward a patient who tends to die is considered one of the most prominent ethical issues in medicine [12]. Family members are critical components in the end-of-life care system due to the dedicated care given to their loved one. During the pandemics, in concern for contagious and disease spread, family members were not allowed to visit their loved ones even on deathbed. Therefore, the separation process of families from the terminally ill patients was different from the previous process we have known and followed. When the patient's condition was deteriorated for the first time, we had to adjust this process in the optimal way within consideration of the existing limitations. At first, the medical teams used digital means such as to place a phone beside the patient's ear to let him make a final conversation with his family. Over time, ethical principles, humanity and compassion values, and moral obligation have led to alternative separation process.

In order to respect the patient who tends to die, as well as to keep the patient's family members safe, we decided to let first-degree relatives to visit their beloved ones while they are with full protection equipment, accompanied by social worker and medical staff, in order to say goodbye and be with their loved ones in their final moments. Studies that examined the effect of separation from terminally ill patients on their family found that treating the patients made them feel satisfied and meaningful, but it also made them feel anxious and depressed [13]. In this new reality of the Corona pandemic, it is still too early to analyse and to understand the consequences of the separation from terminally ill patients on their family members, who experienced an incomplete separation that might have prolong and lifetime influence. 


\section{Emotional support for the teams}

The COVID-19 pandemics have changed world orders for us as individuals, family members and especially as hospital workers. The medical teams were at forefront of fight against the Corona virus. There was no time to express the feelings of anxiety, uncertainty, loss of control and worries for our safety and mainly our loved ones' safety at home. Treating the COVID-19 patients provoked a professional challenge and curiosity that has enhanced the sense of personal responsibility, dedication, meaning, and recognition of the importance of our role as a medical and professional team. The applause of the Israeli people in balconies and airplanes flights in the honor of medical staff members as well as the consistent media coverage, made the medical teams feel that they were playing a vital role in this national mission. All of the above were important factors of resilience that helped to strengthen and maintain the function of the medical teams. At the same time, this period has provoked emotional turmoil and crisis feeling due to prolonged coping with personal and professional uncertainty. Some felt fear of the unknown, the lack of control and even real anxiety from what to come in future. Moreover, insecurity and guilty feelings arose as a result of the change in everyday routine, the isolation from the family, combined with economic and existential concerns causing sleep disturbances and mood disorders among the team members.

The concept of shared trauma appeared in the literature after the Twin towers attack in September 2011, as well as for caregivers coping with patients in Sderot and the Gaza strip. This concept refers to a situation in which both the caregiver and the patient belong to the same community and are being exposed to the same threat at the same time. The shared trauma was found to be extremely relevant for us in this unprecedented situation. The medical staff members, just like the patients, dealt with anxiety and uncertainty feelings [14]. Unlike the patients, health care professionals could experience additional stress during the pandemic. Their challenges included high workload, along with fearing of being infected, treatment of rapidly deteriorating patients, treatment of medical colleagues who got infected, dealing with moral dilemmas, working with new and changing protocols in a rapidly evolving practice environment, along with dealing with ethical conflicts around their roles as healthcare professionals, as parents or as caregivers [15]. In order to maintain the medical team work effective, their mental health condition must be monitored to allow appropriate support interventions when needed. The social workers, who were integral part of the medical teams, recognised the need of emotional support for the medical team members and therefore provided it in an individual and group manner. In these support sessions variety of tools for coping with anxiety and trauma symptoms were provided.

\section{Who is taking care of the caregiver?}

What happened to the treatment when both sides are holding the same scary reality? How can one treat from this supposedly equitable place? The social workers have also experienced difficulties, defences and anxiety, shared reality of uncertainty, lack of control and helplessness. There is wide documentation in the literature of impact of traumatic events on the social workers. Secondary trauma is a process occurring among caregivers of trauma patients, with the caregivers may report experience distress feelings themselves [16]. Indeed, burnout and secondary traumatization are familiar feelings for us as social workers. In this dramatic national crisis, we had to maintain 'self-possession and contemplation' more than ever, in the purpose of building personal resilience as both caregivers and as individuals [17]. Therefore, interpersonal support was emphasized among the medical teams alongside Natal assistance from the Israel trauma and resiliency centre.

\section{Summary}

Suddenly, in the middle of winter 2020, the Corona virus emerged and as if to say for us: 'Stop... from today and further, our lives will never be the same'. We have all been caught into a new and unfamiliar situation, which has changed our lives without recognition. The hospital's social workers team addresses the psychosocial issues of both illness and healthiness conditions. Interventions are based on biopsychosocial broad and multi-systematic assessment, which focuses on the patient's and his family coping with the disease, and finding the appropriate solutions for the patient according to his wishes, culture and values. Despite previous encounters of the staff with varied illness situations and crises, these Corona times required novel preparations of the social workers in the hospital. New issues rose rapidly and the interventions had to be adjusted respectively. The interventions during the COVID-19 pandemic included dealing with patients' loneliness, isolation and anxiety feelings, uncertainty regarding the disease clinical course, supporting the medical teams while working around the clock, and processing the experience and the difficulties of remote care treatments for the patients and their families. Additionally, ethical dilemmas were raised, regarding the patient's rights, participating in the treatment process, choosing hospital discharge destination and end-of life issues. The medi- 
cal social workers vision is: commitment to clients, commitment to professionalism, excellence and partnership in emotional, physical and social aspects. There is no doubt that the team experienced great pride for its professionalism, unique contribution, motivation and creativity. We had the opportunity to make a mark in the pages of history and to be a part of a national mission, alongside the best medical, nursing and para-medical teams. All of the above contributed a special meaning to our role.

'To dream a dream without decipher it, is being equivalent to receiving a letter without opening its envelope', said Freud. The one who will not look, learn, gain insights from the Corona crisis and treat his patients as before, will be as 'the one who walks in the darkness and whistling, thus feeling better but does not see better' (Freud). [18].

\section{References}

1. Jin Y, Yang H, Ji W, et al. Virology, epidemiology, pathogenesis, and control of covid-19. Viruses. 2020;12(4):1-17. doi:10.3390/v12040372

2. Halon E. Israel limits gatherings to 100 people as coronavirus cases climb to 7. Jerusalem Post. 2020. https://www.jpost.com/Israel-News/Coronavirus-cases-climb-to-77-second-case-of-unknown-origin-confirmed-620578

3. Zeidman A, Bathish J, Zabida A, Eisenberg L, Zhalka F, et. Al. Clinical experience of COVID-19 patients: Disease course and treatment features. G Med Sci. 2020; 1(2): 005-015. https://www.doi.org/10.46766/thegms.virology.20060302

4. Davis S. Baldry E. Milosevic B. Walsh A. Defining the role ofr the hospital social worker in Australia. International Social Work. 2004. https://www.doi.org/10.1177/0020872804043958

5. WHO. Coronavirus disease. World Heal Organ. 2020;2019(March):2633. https://www.doi.org/10.1001/ jama.2020.2633

6. Croff P. Clinical social work: Present role and future challenge. Social work in health care. 1988; 13:21-33.

7. Kang L, Li Y, Hu S, Chen M, Yang BS et al : The mental health of medical workers in Wuhan China dealing with the 2019 novel coronavirus. The Lancet Psychiatry, 7(3), e 14.

8. Lima C.K.T, De Mederios Carvalho P.M., Lima I.D.A.S, dEoliviero Nunes et al: The emotional impact of Coronavirus 2019 Ncov(New Coronavirus) Psychiatry research 2020. 1129

9. Zhang J, Wu W, Zhao X, Zhang W: Recommended Psychological crisis intervention response to the novel coronavirus pneumonia outbreak in China: a model of west China hospital. Precision Clinical Medicine 2020. 3:3-8

10. Shanafelt T, Ripp J, Trockel M.: Understanding and addressing source of anxiety among health care professionals during the COVID-19 pandemics.

11. Davidson K. Evolving social work roles in health care: The case of discharge planning. Social work in health care. 1978; 4:43-76

12. Romo RD. Allison TA, Smith AK. Wallhagen MI. Sense of control in End of Life decision making. J of AMERICAN Geriatric Society. 2017. 65(3);70-75.

13. Erickson KA. How we die now: intimacy and the work of dying. Temple University Press. 108-112

14. Dan L. Longo. Blinderman, C.D. Billings, JA. Comfort Care for Patients Dying in the Hospital. N Engl J Med 2015; 373: 2549-61. DOI: 0.1056/NEJMra1411746

15. Walton M: Mental health care for medical staff and affiliated healthcare workers during the COVID-19 pandemic. European Heart Journal. 2020.

16. Sullivan AB. Miller D. Who is taking care of the caregiver. J Patient Experience. 2015. 2;712

17. Adelman RD. Tmanova LL. DeLegado D. Dion S. Lachs M. Caregiver burden: A clinical review. JAMA. 2014.311;105260.

18. Shalev Arie: “Corona, Where to?”. Talking at corona times. Sihot J of Israeli Psychotherapy. \{Hebrew\}. April 2020. 